\title{
Terahertz emission mediated by ultrafast time-varying metasurfaces
}

\author{
J. Tunesi $\odot,{ }^{1,{ }^{*}}$ L. Peters $\odot,{ }^{1}$ J. S. Totero Gongora $\odot,{ }^{1}$ L. Olivieri $\odot,{ }^{1}$ A. Fratalocchi, ${ }^{2}$ A. Pasquazi, ${ }^{1}$ and M. Peccianti $\odot$ 1, \\ ${ }^{1}$ Emergent Photonics Laboratory (EPic), Department of Physics and Astronomy, University of Sussex, Brighton BN1 9QH, United Kingdom \\ ${ }^{2}$ PRIMALIGHT, King Abdullah University of Science and Technology (KAUST), Thuwal 23955-6900, Saudi Arabia
}

(Received 16 August 2021; accepted 16 September 2021; published 18 October 2021)

\begin{abstract}
Systems with ultrafast time-varying dielectric properties represent an emerging physical framework. We demonstrate here the observation of subcycle dynamics interacting directly with an electromagnetic source comprised of morphologically constrained photoexcited carriers in a surface nanostructure. A transition to a metallic metasurface state occurs on time scales faster than the terahertz-field period, inducing large nonlinear ultrafast phase shifts in the terahertz emission and exposing an interesting physical setting.
\end{abstract}

DOI: 10.1103/PhysRevResearch.3.L042006

Materials with time-varying physical properties provide an attractive framework to study different forms of nonlinear light-matter interactions [1-4]. In these systems, an electric field propagating in the medium perceives a temporal variation in the dielectric properties of the medium as a temporal boundary $[5,6]$. In the presence of a temporal discontinuity of the refractive index between two values $n_{i}$ and $n_{f}$, the conservation of the electromagnetic field components leads to the definition of "temporal" refraction and reflection laws affecting the energy and spectral properties of the waves and involving the transition of the frequency from $\omega_{i}$ to $\omega_{f}$ via $n_{i} \omega_{i}=n_{f} \omega_{f}$ [7-9]. By taking advantage of this relationship, it is possible to directly modify the spatiotemporal properties of the electromagnetic wave and induce exotic nonlinear responses such as time refraction and photon acceleration [8,10-12]. These phenomena have also been applied to achieve adiabatic frequency conversion [13-15], antireflection waveguide couplers [16], spontaneous photon emission [17] and dynamical tuning of the Poynting vector direction [18]. The development of new investigative tools to study time-dependent media is also attracting significant attention in the context of epsilon-near-zero media. In these systems, the combination of tunable material response and high nonlinearity is responsible for large frequency shifts in electromagnetic waves scattered from temporal boundaries [19-21]. In this context, the absorption of ultrashort optical pulses in semiconductor nanostructures and metamaterials provides an ideal framework to study time-dependent optical responses in accessible experimental settings [22,23]. Due to the optical injection of a large density of excited photocarriers, the material undergoes an ultrafast metallization process

\footnotetext{
*j.tunesi@sussex.ac.uk

${ }^{\dagger}$ m.peccianti@sussex.ac.uk
}

Published by the American Physical Society under the terms of the Creative Commons Attribution 4.0 International license. Further distribution of this work must maintain attribution to the author(s) and the published article's title, journal citation, and DOI. corresponding to a time-dependent complex susceptibility [24,25]. Such an effect is analogous to the flash ionization of gases in optical cavities, where the ultrafast formation of plasma leads to the onset of complex interactions among the optical waves [26]. When the dielectric transient occurs within time scales shorter than the optical period, the mismatch between the characteristic time scales of photonic responses and their coupling with the propagating fields poses a significant challenge. This condition is met in the framework of terahertz (THz) pulse generation by photoexcited carrier dynamics. The typical $\mathrm{THz}$ period $(\sim 1 \mathrm{ps})$ can be significantly longer than the metallization transient induced by standard ultrafast optical sources ( $\sim 100$-fs pulses $)$. Additionally, the investigation of time-dependent effects with $\mathrm{THz}$ pulses enables the intriguing possibility to perform fully coherent field-sensitive detection, as in the case of time domain spectroscopy (TDS). TDS techniques enable the time-resolved detection of the field oscillations and retrieve any variation in the amplitude and phase of the $\mathrm{THz}$ pulse as its source interacts with the time-varying medium [27]. TDS detection, moreover, could provide the ability to perceive fast-arising time boundaries beyond the effect they produce on the power spectral density distribution of the electromagnetic wave [5,28,29]. In the past few years, this possibility has been explored in a series of optical pump terahertz probe (OPTP) spectroscopy experiments focused on the direct observation of the impact of rapid variations of the density of free carriers on the $\mathrm{THz}$ electric field [30-32]. Quite interestingly, for transitions much faster than the $\mathrm{THz}$ carrier period, researchers have observed the formation of additional frequencies in the $\mathrm{THz}$ pulse due to the onset of $\mathrm{THz}$ shockwaves reflected by an ultrashort step in the conductivity [28]. In these experiments, however, the ultrafast transient simply clips the $\mathrm{THz}$ wave form in time, and the new frequencies actually result from ultrafast damping of the dynamics. As in the case of standard temporal refraction in continuous wave $(\mathrm{CW})$ systems, no energy transfer takes place between the pump and probe pulses, and the energy at these new frequencies is simply redistributed from the original spectral profile [13,33]. More importantly, in these conditions, the propagating wave interacts with a spatially static 
(a)

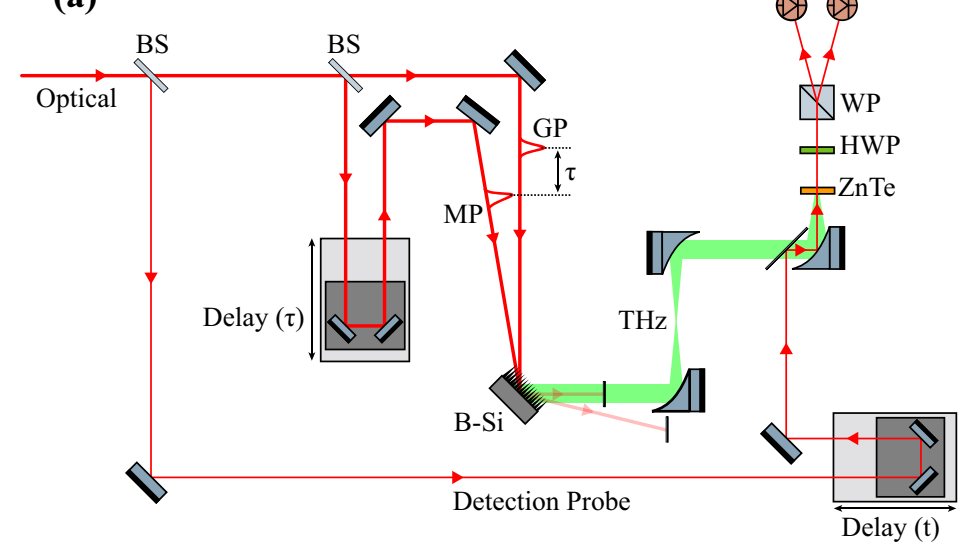

(b)
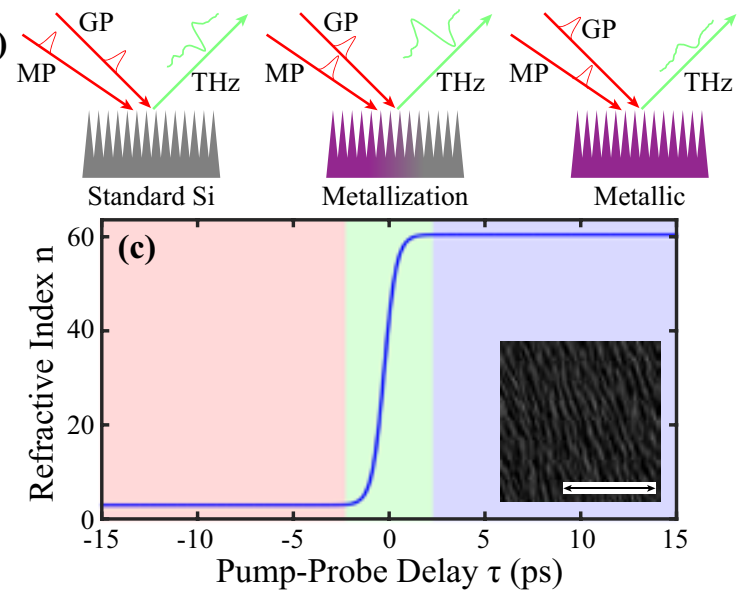

FIG. 1. (a) The full experimental setup for $\mathrm{THz}$ generation and ultrafast metallization of black silicon substrates. BS: beam splitter, GP: generation pump, MP: metallization pump, $B$-Si: black silicon, ZnTe: zinc telluride, HWP: half-wave plate, WP: Wollaston prism. (b) Schematic illustration of the three major regimes in the transient measurements as the $B$-Si transitions from a semiconducting to a metallic state. (c) The corresponding shift in the real part of the time-dependent refractive index induced by the MP. The inset shows an SEM image of the $B$-Si surface (scale bar $5 \mu \mathrm{m}$ ).

temporal conductivity transient, and the modulation is critically smeared in time by the large velocity mismatch between the modulating mechanism and the propagating wave. An open question is then how an ultrafast temporal boundary can affect the spatiotemporal properties of nonlinear sources localized within the medium.

In this work, we investigate a physical scenario in which a nonlinear $\mathrm{THz}$ source lies within an ultrafast time-varying medium. To this end, we employ a black silicon $(B-\mathrm{Si})$ material platform consisting of a random distribution of $\mathrm{Si}$ nanopillars [34], with subwavelength average spacing. The $B$-Si metasurface structure acts to create a smooth transition in the effective refractive index in the direction perpendicular to the surface, creating an impedance matching interface and thus highly reducing its reflectivity (below 10\%) across the visible spectral range [34,35]. As $B$-Si is known to emit $\mathrm{THz}$ radiation when excited by optical pulses [36], this platform allows us to observe the effects of the ultrafast metallization on a concurrent $\mathrm{THz}$ generation mechanism. Although the precise mechanics of $\mathrm{THz}$ emission from $B$-Si is still a matter of debate in the literature, it is widely accepted that the morphological confinement of the carrier dynamics plays a primary role due to the exclusively $p$-polarized radiated field and the fact that silicon surfaces inherently do not support relevant generation mechanisms [36,37]. In a phenomenological model, a morphologically constrained transition to a metallic metasurface sustains currents in the pillars that represent a distribution of weakly resonant, dipolelike coupled radiators [38-40]. Microscopically, the system behaves as an effective nonlinear medium supporting a field-matter interaction partially resembling dynamics observed in photo-Dember generation and surface-field driven surge-current effects. The local generation is then dependent on the morphological parameters such as the aspect ratio, length, and spatial distribution density. This nanopillar metasurface structure plays simultaneous roles in both significantly reducing the absorption length and inducing a surface symmetry breaking, which enables ponderomotive forces to act on the photocarriers. We investigated the effect of the ultrafast metallization on the $\mathrm{THz}$ generation through a dual-pump optical setup inspired by the optical pump rectification emission (OPRE) scheme discussed thoroughly in $[41,42]$. OPRE is a nonlinear pump-probe technique designed to capture the interaction of $\mathrm{THz}$ generation mechanisms as a function of the photocarrier dynamics in the sample. By characterizing the emitted $\mathrm{THz}$ field at the different stages of the metallization process, we unveil the onset of complex phase and amplitude dynamics originating from the nonlinear interaction between temporal boundaries and $\mathrm{THz}$ sources. Our results, confirmed by numerical simulations, open the realization of a different class of tunable $\mathrm{THz}$ sources based on time-dependent nonlinear metamaterials. Our experimental setup is illustrated in Fig. 1(a). We employed a regenerative-amplified ultrafast source (Coherent Libra $\mathrm{HE}+$ ) which provides a train of ultrashort pulses with $1 \mathrm{kHz}$ repetition rate, $800 \mathrm{~nm}$ central wavelength, $100 \mathrm{fs}$ pulse duration, and a beam diameter at $e^{-2}$ of $9 \mathrm{~mm}$. We split the beam into three lines. The two main lines are responsible for the generation of $\mathrm{THz}$ (generation pump, $1.5 \mathrm{~mJ}$ ) and to induce the ultrafast metallization in the sample (metallization pump, $2.5 \mathrm{~mJ}$ ). We controlled the delay between the two pumps by means of a mechanical translation stage. The third line $(\sim 1 \mu \mathrm{J})$ acted as an optical probe for the detection of the generated $\mathrm{THz}$ field via electro-optic sampling [43]. In our experiments, we confirmed that no artifacts are introduced by the implementation of the strong generation pump by ensuring that the angle between the pumps is large enough $\left(\sim 11.9^{\circ}\right)$ to accurately spatially discriminate the $\mathrm{THz}$ fields generated by both pumps or the cross products between them. We fabricated our $B$-Si samples through a self-masking inductively coupled plasma-reactive ion etching (ICP-RIE) protocol in a $\mathrm{SF}_{6} / 0_{2}$ plasma [44]. In this procedure, the competition between surface passivation, ion bombardment, and chemical etching leads to a highly anisotropic etching which results in the spontaneous formation of sharp silicon needles on the surface [34]. The resulting morphology is shown in the inset of Fig. 1(c), with average needle heights on the order of 

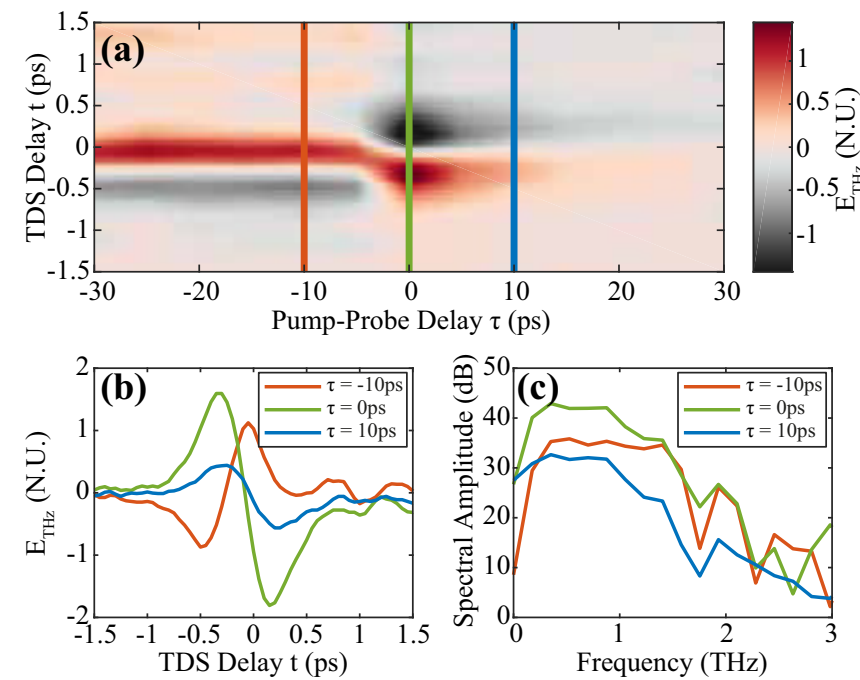

FIG. 2. (a) The full transient $\mathrm{THz}$ dynamics spanning tens of picoseconds before and after photoexcitation. (b) The $B-\mathrm{Si} \mathrm{THz}$ wave forms taken at the pump-probe delay times depicted by the lines marked in (a). With $\tau=-10 \mathrm{ps}, \tau=0 \mathrm{ps}$, and $\tau=10 \mathrm{ps}$ corresponding to the standard unexcited $B$-Si, the transient metallization region, and the metallic state respectively. (c) The THz spectra obtained via the fast Fourier transform of the temporal wave forms shown in (b).

$1.6 \mu \mathrm{m}$ and apex angles $\sim 12^{\circ}$. The generation pump induces a morphologically driven photocurrent $\mathbf{J}(t)$, dependent on the impinging pulse energy, which acts as a direct $\mathrm{THz}$ source:

$$
\mathbf{E}_{\mathrm{THz}}(t) \propto \frac{\partial \mathbf{J}(t)}{\partial t} .
$$

The metallization pump, conversely, drives an ultrafast metallization transient, i.e., a temporal variation in the free-carrier density $N(t)$ :

$$
N(t)=[H(t-\tau) * A(t-\tau)] e^{-(t-\tau) / \tau_{\mathrm{Si}}},
$$

where $\tau$ is the intrapump delay, $H(t)$ is the Heaviside step function, $A(t-\tau)$ is the pump pulse envelope, and $\tau_{S i}(>1 \mathrm{~ns})$ is the characteristic carrier recombination time (we assume the rising front of the metallization is instantaneous in the time scale of the $\mathrm{THz}$ wave period). By acting on the delay $\tau$ between the two optical pumps, we could observe the effects of the temporal discontinuity on the $\mathrm{THz}$ emission across all the different stages of the metallization process. As illustrated in the temporal trace shown in Fig. 2, we observed the onset of rather different and counterintuitive regimes in the emitted far-field $\mathrm{THz}$ wave form. When $\tau<-5 \mathrm{ps}$, the generation pulse reaches the sample before the metallization pulse. In this condition, the generated $\mathrm{THz}$ field corresponds to the standard emission from $B$-Si substrates that originates from photocarrier drift-diffusion mechanisms [Fig. 2(b), red line] [36]. At $\tau \approx-5 \mathrm{ps}$, the generation and metallization pulses begin overlapping in the sample, where the ultrafast injection of photocarriers leads to a rapid change in the complex dielectric function in proximity to the $\mathrm{THz}$ source. Quite interestingly, the effect of the ultrafast metallization on the $\mathrm{THz}$ emission manifests through a stark coherent phase shift and enhancement of the generated pulse. This effect peaks at $\tau=0$ ps [Fig. 2(b), green line]. In this scenario, the conductivity transient acts as a temporal boundary that is perfectly overlapped in space and synchronized in time with the THz source, causing a shift in the position of the peak of the detected $\mathrm{THz}$ field. It should be noted that the observed delayed onset of the shift, which is maximized at $\tau=0 \mathrm{ps,}$ is a consequence of the finite angle between generation and metallization pumps, which results in unavoidable temporal smearing in the measurement (on the scale of a few ps). However, locally at each surface $\mathrm{THz}$ source point, the effect is always driven on the time scale of the optical pulse duration. After the transition is completed ( $\tau>5 \mathrm{ps}$ ), the system relaxes to a stationary condition following two notable time scales. At first, the field enhancement decays quite rapidly as the temporal overlap between the probe and the index step increases within the smearing. Second, the system follows a slow recovery dynamic due to the spontaneous recombination of photocarriers in the sample. Notably, the phase shift is preserved even after the temporal transition is complete, suggesting that it is fundamentally related to the onset of a metallic state in the $B$-Si needles. In our experiments, we noticed that the magnitude of the phase shift is independent of the pumping rate but varies significantly across samples with different morphologies, suggesting that the effect is tailored by the specific geometry of the $B$-Si nanopillars. Furthermore, we observed that the amplitude could be tuned by acting on several pumping parameters such as wavelength, polarization, and pulse energy, which modify the photocarrier density, energy, and spatial overlap (through the variation of skin depths and absorption profiles at different wavelengths) with the interacting fields, suggesting local and group resonances in the field-matter interaction.

To provide further insights on the fundamental process driving the phase shift, we studied the ultrafast metallization process through fully dispersive 1D finite-differences in time domain (FDTD) simulations, where material dispersion was implemented via an auxiliary differential equation approach [45]. More specifically, we integrated a time-varying material configuration by generalising a standard Drude model to include time-dependent variations. Following standard approaches, we expressed the Drude dielectric function as follows:

$$
\epsilon(\omega, t-\tau)=\epsilon_{\infty}-\frac{\left[2 \pi v_{p}(t-\tau)\right]^{2}}{\omega^{2}-i \omega \gamma(t-\tau)},
$$

where $\epsilon_{\infty}$ is the high-frequency dielectric constant [46]. In Eq. (3), we explicitly introduced the time-dependent properties of the medium through the scattering rate $\gamma(t-\tau)$ and the material's plasma frequency $v_{p}(t-\tau)$. The first is determined by a combination of electron-electron scattering and electronphonon scattering contributions, while the latter is expressed in a semiconductor as

$$
v_{p}(t-\tau)=\frac{1}{2 \pi} \sqrt{\frac{e^{2} N(t-\tau)}{m^{*} \epsilon_{0} \epsilon_{\infty}}},
$$

where $e, m^{*}, \epsilon_{0}$ denote the electron charge, effective mass, and vacuum permittivity, respectively. In our simulations, we assumed a plasma frequency of $v_{p}=0.75 \mathrm{THz}$ for the stationary 

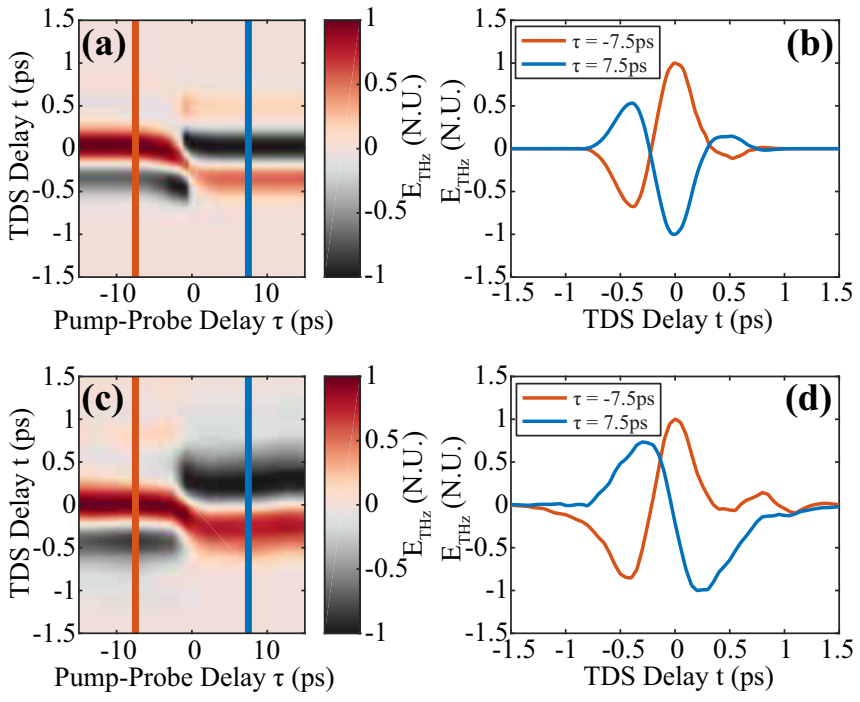

FIG. 3. (a) The fully simulated THz time-dependent dynamics, where all wave forms have been individually normalized to highlight the phase shift induced by the material metallization. (b) The temporal $\mathrm{THz}$ wave forms corresponding to the pump-probe delay times marked in (a), with $\tau=-7.5$ ps and $\tau=7.5$ ps being the semiconducting and metallic states, respectively. (c) The experimental dynamics shown in Fig. 2 with the same normalization to demonstrate the behavior similar to that shown in (a). (d) The experimental wave forms taken at the same values of the delay $\tau$ as in (b).

case and $v_{p}=70 \mathrm{THz}$ for the photoexcited case, corresponding to an excitation free-carrier density of $2.6 \times 10^{20} \mathrm{~cm}^{-3}$ compatible with our experimental conditions. Following the approach described in Ref. [47], we also assumed a scattering rate of $\frac{1}{0.8 \mathrm{fs}}$ before excitation and $\frac{1}{10.5 \mathrm{fs}}$ upon excitation due to reduced electron scattering in the higher conductivity regime [48]. In modeling this time-dependent plasma frequency, we have assumed that any additional modification to the effective mass caused by the metallization pump is a second-order effect and can be considered constant in this study. Our FDTD simulations consisted of a $\mathrm{THz}$ pulse source launched from within a Si substrate whose dielectric function is varying as given by Eqs. (2)-(4) for different values of the intrapump delay $\tau$. We considered a slightly longer excitation pulse duration of $640 \mathrm{fs}$ to minimize the onset of spurious reflections in the FDTD propagator [45]. The model accounts for the experimental temporal smearing with a low-pass filter (with a time constant of $3 \mathrm{ps}$ ) along with the pump-probe delay axis $\tau$, to directly translate the ultrafast temporal response calculated by the FDTD propagator onto the expected physical response that would be observed in our experimental condition without modifying the time-dependent optical processes being investigated. Remarkably, our numerical simulations can reproduce the experimental phase shift accurately, as shown in Fig. 3, where we compare a typical result of the simulations with our experiments. Such a relation provides core evidence that our photoexcited metasurface mainly behaves as a temporally varying material undergoing an ultrafast metallization. Specifically, our choice of material parameters corresponds to a refractive index discontinuity of $\sim 57$, consistent with the magnitude normally achievable in plasmonic systems. This suggests that the temporal boundary is mediated, in fact, by surface plasmon polariton (SPP) waves [48-50] enabled by the nanopillar geometry, where a jump in refractive index can be much larger than the planar case. In fact, such plasmonic confinement in the pillars could reveal insights into the origin of the surprisingly strong $\mathrm{THz}$ emission from $B-\mathrm{Si}$ surfaces, where the morphological confinement and subsequent carrier dynamics, driving an ultrafast $\mathrm{THz}$ photocurrent, are dominated by the excitation of plasmonic modes in the pillars. It is worth noting, however, that the morphology of the photoexcited pillars is only included in terms of their ponderomotive action sustaining the photocurrent transients acting as the source of the terahertz emission. As a result, any potential resonant and localized plasmonic effects induced by the metallic cones or nonlinear mixing cannot be appreciated here. Such effects possibly contribute to higher-order effects, such as the field enhancement observed in Fig. 2, but are remarkably not necessary to reproduce the fundamental phase dynamics of the phenomenon. Finally, it is quite interesting that the plasma frequency rapidly changes in the metallization transient within the frequency band of the generated radiation. This suggests an intriguing connection with the typical conditions of recent observations of enhanced nonlinear conversion in epsilon-near-zero systems [20,21].

In conclusion, we have provided experimental characterization of the effects of an optically induced, ultrafast temporal discontinuity on the field generated by a nonlinear $\mathrm{THz}$ source. This physical scenario is significantly different from previous investigations as the nonlinear source is embedded in the time-varying nanostructure, where the transition to a metallic state and the morphology are at the origin of the dipole-array emission. This physical framework enables the observation of a coherent time-dependent phase-shifting dynamic in the emission and a net gain of the optical-to-terahertz conversion efficiency. The phase-inversion dynamics can be fully traced to the time-dependent transition in the medium, as demonstrated by introducing a time-dependent Drude susceptibility model in FDTD simulations. The observed dependence on the different excitation and morphological parameters on the ultrafast transient behaviors suggests that morphology-driven plasmonic resonances play a key role in the $\mathrm{THz}$ generation mechanism. More fundamentally, our results suggest that inducing a time-dependent dielectric constant within nonlinear optical sources can enable intriguing features. For example, while photocarriers are traditionally only exploited to screen a $\mathrm{THz}$ field in a standard pump-probe embodiment [37], we demonstrated that a time-varying photoexcitation could control the far-field $\mathrm{THz}$ phase by acting solely on the pump-probe delay. By allowing direct access to the amplitude and phase properties of the emitted $\mathrm{THz}$ pulse, we expect that this physical framework could trigger perspectives and methodologies to gain full-field control on the $\mathrm{THz}$ emission while simultaneously providing a benchmark for further investigations on time-dependent phenomena exploiting the optical excitation of $\mathrm{THz}$ field sources. These results provide the base for which future studies, including the full drift-diffusion dynamics of the photocarriers in combination with FDTD modeling, will reveal the full recovery of the experimental system. Furthermore, we predict that such schemes can also create impact in complex nonlinear imaging 
[51,52], THz surface emitters [53], and in the optical control of electron-electron interactions $[54,55]$.

All source data for the plots are available online [56].

J.T. acknowledges the support of the Engineering and Physical Sciences Research Council (EPSRC) through Studentship No. EP/N509784/1. J.S.T.G. acknowledges funding from the Leverhulme Trust (Leverhulme Early Career Fellowship No. ECF-2020-537). A.P. acknowledges the support of the Engineering and Physical Research Council (EPSRC), Industrial Innovation Fellowship Programme, under Grant No. $\mathrm{EP} / \mathrm{S} 001018 / 1$. This project received funding from the European Research Council (ERC) under the European Union's Horizon 2020 Research and Innovation Programme Grant Agreements No. 725046 and No. 851758.
[1] Y. Xiao, D. N. Maywar, and G. P. Agrawal, Reflection and transmission of electromagnetic waves at a temporal boundary, Opt. Lett. 39, 574 (2014).

[2] J. S. Martínez-Romero, O. M. Becerra-Fuentes, and P. Halevi, Temporal photonic crystals with modulations of both permittivty and permeability, Phys. Rev. A 93, 063813 (2016).

[3] V. Bacot, M. Labousse, A. Eddi, M. Fink, and E. Fort, Time reversal and holography with spacetime transformations, Nat. Phys. 12, 972 (2016).

[4] D. M. Solís and N. Engheta, Functional analysis of the polarization response in linear time-varying media: A generalization of the Kramers-Kronig relations, Phys. Rev. B 103, 144303 (2021).

[5] A. Rogov and E. Narimanov, Space-time metamaterials, ACS Photonics 5, 2868 (2018).

[6] I. Liberal, Y. Li, and N. Engheta, Reconfigurable epsilon-nearzero metasurfaces via photonic doping, Nanophotonics 7, 1117 (2018).

[7] R. Fante, Transmission of electromagnetic waves into timevarying media, IEEE Trans. Antennas Propag. 19, 417 (1971).

[8] J. T. Mendonça, Theory of Photon Acceleration, Series in plasma physics (Institute of Physics, Bristol, UK; Philadelphia, PA, 2001).

[9] C. Caloz and Z.-L. Deck-Leger, Spacetime metamaterials-part II: Theory and applications, IEEE Trans. Antennas Propag. 68, 1583 (2020).

[10] M. Cirone, K. Rzazewski, and J. Mostowski, Photon generation by time-dependent dielectric: A soluble model, Phys. Rev. A 55, 62 (1997).

[11] S. Vezzoli, V. Bruno, C. DeVault, T. Roger, V. M. Shalaev, A. Boltasseva, M. Ferrera, M. Clerici, A. Dubietis, and D. Faccio, Optical Time Reversal From Time-Dependent Epsilon-NearZero Media, Phys. Rev. Lett. 120, 043902 (2018).

[12] H. Wu, X. X. Gao, L. Zhang, G. D. Bai, Q. Cheng, L. Li, and T. J. Cui, Harmonic information transitions of spatiotemporal metasurfaces, Light Sci. Appl. 9, 198 (2020).

[13] M. Notomi and S. Mitsugi, Wavelength conversion via dynamic refractive index tuning of a cavity, Phys. Rev. A 73, 051803 (2006).

[14] E. J. Reed, M. Soljačić, and J. D. Joannopoulos, Color of Shock Waves in Photonic Crystals, Phys. Rev. Lett. 90, 203904 (2003).

[15] X. Zhou, S. K. Gupta, X. Zhu, G. Su, P. Zhan, Y. Liu, Z. Chen, M. Lu, and Z. Wang, Nonreciprocal Isolation And Wavelength Conversion Via A Spatiotemporally Engineered Cascaded Cavity, Phys. Rev. Appl. 13, 044037 (2020).

[16] V. Pacheco-Peña and N. Engheta, Antireflection temporal coatings, Optica 7, 323 (2020).
[17] F. Belgiorno, S. L. Cacciatori, M. Clerici, V. Gorini, G. Ortenzi, L. Rizzi, E. Rubino, V. G. Sala, and D. Faccio, Hawking Radiation From Ultrashort Laser Pulse Filaments, Phys. Rev. Lett. 105, 203901 (2010).

[18] V. Pacheco-Peña and N. Engheta, Temporal aiming, Light Sci. Appl. 9, 129 (2020).

[19] V. Bruno, S. Vezzoli, C. DeVault, E. Carnemolla, M. Ferrera A. Boltasseva, V. M. Shalaev, D. Faccio, and M. Clerici, Broad frequency shift of parametric processes in epsilon-near-zero time-varying media, Appl. Sci. 10, 1318 (2020).

[20] J. B. Khurgin, M. Clerici, V. Bruno, L. Caspani, C. DeVault, J. Kim, A. Shaltout, A. Boltasseva, V. M. Shalaev, M. Ferrera, D. Faccio, and N. Kinsey, Adiabatic frequency shifting in epsilonnear-zero materials: the role of group velocity, Optica 7, 226 (2020).

[21] Y. Zhou, M. Z. Alam, M. Karimi, J. Upham, O. Reshef, C. Liu, A. E. Willner, and R. W. Boyd, Broadband frequency translation through time refraction in an epsilon-near-zero material, Nat. Commun. 11, 2180 (2020).

[22] S. Han, L. Cong, Y. K. Srivastava, B. Qiang, M. V. Rybin, A. Kumar, R. Jain, W. X. Lim, V. G. Achanta, S. S. Prabhu, Q. J. Wang, Y. S. Kivshar, and R. Singh, All-dielectric active terahertz photonics driven by bound states in the continuum, Adv. Mater. 31, 1901921 (2019).

[23] K. Kondo and T. Baba, Adiabatic wavelength redshift by dynamic carrier depletion using $\mathrm{p}$ - $\mathrm{i}$ - $\mathrm{n}$-diode-loaded photonic crystal waveguides, Phys. Rev. A 97, 033818 (2018).

[24] A. J. Sabbah and D. M. Riffe, Femtosecond pump-probe reflectivity study of silicon carrier dynamics, Phys. Rev. B 66, 165217 (2002).

[25] M. C. Beard, G. M. Turner, and C. A. Schmuttenmaer, Transient photoconductivity in GaAs as measured by time-resolved terahertz spectroscopy, Phys. Rev. B 62, 15764 (2000).

[26] A. B. Shvartsburg, Optics of nonstationary media, Phys. Usp. 48, 797 (2005).

[27] S. L. Dexheimer, Terahertz Spectroscopy: Principles and Applications (CRC, Boca Raton, 2007).

[28] J. Bühler, J. Allerbeck, G. Fitzky, D. Brida, and A. Leitenstorfer, Terahertz shockwaves generated by a precise subcycle cut of the electric field, Optica 5, 821 (2018).

[29] K. Lee, J. Son, J. Park, B. Kang, W. Jeon, F. Rotermund, and B. Min, Linear frequency conversion via sudden merging of meta-atoms in time-variant metasurfaces, Nat. Photonics $\mathbf{1 2}$ 765 (2018)

[30] M. C. Nuss, D. H. Auston, and F. Capasso, Direct Subpicosecond Measurement of Carrier Mobility of Photoexcited Electrons in Gallium Arsenide, Phys. Rev. Lett. 58, 2355 (1987). 
[31] C. A. Schmuttenmaer, Exploring dynamics in the far-infrared with terahertz spectroscopy, Chem. Rev. 104, 1759 (2004).

[32] P. Uhd Jepsen, W. Schairer, I. H. Libon, U. Lemmer, N. E. Hecker, M. Birkholz, K. Lips, and M. Schall, Ultrafast carrier trapping in microcrystalline silicon observed in optical pump-terahertz probe measurements, Appl. Phys. Lett. 79, 1291 (2001).

[33] X. Zhou, S. K. Gupta, G. Su, P. Zhan, M. Lu, and Z. Wang, Multiple-wavelength conversion based on the anomalous Doppler effect induced by dynamic tuning in a self-collimation photonic crystal, Phys. Rev. A 100, 013830 (2019).

[34] X. Liu, P. R. Coxon, M. Peters, B. Hoex, J. M. Cole, and D. J. Fray, Black silicon: fabrication methods, properties and solar energy applications, Energy Environ. Sci. 7, 3223 (2014).

[35] C. Wu, C. H. Crouch, L. Zhao, J. E. Carey, R. Younkin, J. A. Levinson, E. Mazur, R. M. Farrell, P. Gothoskar, and A. Karger, Near-unity below-band-gap absorption by microstructured silicon, Appl. Phys. Lett. 78, 1850 (2001).

[36] P. Hoyer, M. Theuer, R. Beigang, and E.-B. Kley, Terahertz emission from black silicon, Appl. Phys. Lett. 93, 091106 (2008).

[37] U. Blumröder, M. Zilk, H. Hempel, P. Hoyer, T. Pertsch, R. Eichberger, T. Unold, and S. Nolte, Influence of structure geometry on $\mathrm{THz}$ emission from black silicon surfaces fabricated by reactive ion etching, Opt. Express 25, 6604 (2017).

[38] P. Bharadwaj, B. Deutsch, and L. Novotny, Optical antennas, Adv. Opt. Photonics 1, 438 (2009).

[39] R. M. Córdova-Castro, A. V. Krasavin, M. E. Nasir, A. V. Zayats, and W. Dickson, Nanocone-based plasmonic metamaterials, Nanotechnology 30, 055301 (2019).

[40] D. V. Seletskiy, M. P. Hasselbeck, J. G. Cederberg, A. Katzenmeyer, M. E. Toimil-Molares, F. Léonard, A. A. Talin, and M. Sheik-Bahae, Efficient terahertz emission from InAs nanowires, Phys. Rev. B 84, 115421 (2011).

[41] L. Peters, J. Tunesi, A. Pasquazi, and M. Peccianti, Optical pump rectification emission: route to terahertz free-standing surface potential diagnostics, Sci. Rep. 7, 9805 (2017).

[42] L. Peters, J. Tunesi, A. Pasquazi, and M. Peccianti, High-energy terahertz surface optical rectification, Nano Energy 46, 128 (2018).

[43] Q. Wu and X. C. Zhang, Ultrafast electro-optic field sensors, Appl. Phys. Lett. 68, 1604 (1996).
[44] H. Jansen, M. de Boer, R. Legtenberg, and M. Elwenspoek, The black silicon method: a universal method for determining the parameter setting of a fluorine-based reactive ion etcher in deep silicon trench etching with profile control, J. Micromech. Microeng. 5, 115 (1995).

[45] A. Taflove and S. C. Hagness, Computational Electrodynamics: The Finite-difference Time-domain Method, 3rd ed. (Artech House, Boston, 2005).

[46] S. A. Maier, Plasmonics: Fundamentals and Applications (Springer, New York, 2007).

[47] G. Della Valle, B. Hopkins, L. Ganzer, T. Stoll, M. Rahmani, S. Longhi, Y. S. Kivshar, C. De Angelis, D. N. Neshev, and G. Cerullo, Nonlinear anisotropic dielectric metasurfaces for ultrafast nanophotonics, ACS Photonics 4, 2129 (2017).

[48] M. I. Stockman, Plasmonics: Theory and Applications (Springer, New York, 2013).

[49] D. K. Gramotnev and S. I. Bozhevolnyi, Plasmonics beyond the diffraction limit, Nat. Photonics 4, 83 (2010).

[50] A. V. Zayats, I. I. Smolyaninov, and A. A. Maradudin, Nanooptics of surface plasmon polaritons, Phys. Rep. 408, 131 (2005).

[51] L. Olivieri, J. S. Totero Gongora, A. Pasquazi, and M. Peccianti, Time-resolved nonlinear ghost imaging, ACS Photonics 5, 3379 (2018).

[52] L. Olivieri, J. S. T. Gongora, L. Peters, V. Cecconi, A. Cutrona, J. Tunesi, R. Tucker, A. Pasquazi, and M. Peccianti, Hyperspectral terahertz microscopy via nonlinear ghost imaging, Optica 7, 186 (2020)

[53] J. S. Totero Gongora, L. Peters, J. Tunesi, V. Cecconi, M. Clerici, A. Pasquazi, and M. Peccianti, All-Optical Two-Color Terahertz Emission From Quasi-2D Nonlinear Surfaces, Phys. Rev. Lett. 125, 263901 (2020).

[54] E. A. Nanni, W. R. Huang, K.-H. Hong, K. Ravi, A. Fallahi, G. Moriena, R. J. Dwayne Miller, and F. X. Kärtner, Terahertzdriven linear electron acceleration, Nat. Commun. 6, 8486 (2015).

[55] D. A. Walsh, D. S. Lake, E. W. Snedden, M. J. Cliffe, D. M. Graham, and S. P. Jamison, Demonstration of sub-luminal propagation of single-cycle terahertz pulses for particle acceleration, Nat. Commun. 8, 421 (2017).

[56] DOI: $10.25377 /$ sussex.16732690. 